\title{
Dissecção Aguda de Aorta Ascendente e de Tronco de Coronária Esquerda Durante Implante Eletivo de Stents Coronários
}

\author{
Esmeralci Ferreira ${ }^{1,2,3,4,5}$, Alcides Ferreira Jr. ${ }^{1,2,5}$, Cyro Vargues Rodrigues ${ }^{1,2,5}$, André Valentim ${ }^{1}$, \\ José Geraldo Amino ${ }^{6}$, Jose Ricardo Pimentel Palazzo ${ }^{1}$, Camillo de Lellis Carneiro Junqueira ${ }^{2,5}$, \\ Guilherme Nossar ${ }^{2,5}$, Valter Gabriel Maluly ${ }^{5}$, Bernardo Amorim ${ }^{1}$
}

\section{RESUMO}

Paciente com angina pós-infarto, associada a lesões graves, em artérias circunflexa e marginal esquerda. Foi submetida a angioplastia com implante de stents, mas apresentou grave dissecção do tronco de coronária esquerda (TCE), que se estendeu para o segmento ascendente da aorta, evoluindo com hipotensão, parada cardiorrespiratória e posterior instabilidade hemodinâmica. Houve resolução imediata do quadro clínico com implante de stent no TCE.

DESCRITORES: Aneurisma dissecante. Angioplastia/efeitos adversos. Stents/efeitos adversos.

A dissecção iatrogênica da aorta ascendente é uma complicação incomum, mas potencialmente catastrófica, das intervenções vasculares. Quando associada ao cateterismo cardíaco, é mais frequentemente diagnosticada como uma impregnação radiopaca na parede aórtica após a injeção de contraste no estudo angiográfico. O principal mecanismo envolvido é a extensão retrógrada de uma dissecção coronária em direção ao arco aórtico e seus ramos. Paralelamente, em cerca de $50 \%$ dos $\operatorname{casos}^{1}$, a dissecção evolui de forma anterógrada, envolvendo a árvore coronária e eventualmente ocasionando oclusões

\footnotetext{
1 Clínica Status Cor - Rio de Janeiro, RJ, Brasil.

2 Hospital de Clínicas Mario Lioni - Duque de Caxias, RJ, Brasil.

${ }^{3}$ Hospital Prontocor - Rio de Janeiro, RJ, Brasil.

${ }^{4}$ Universidade do Estado do Rio de Janeiro (UERJ) - Rio de Janeiro, RJ, Brasil.

${ }_{5}$ Universidade do Grande Rio (Unigranrio) - Duque de Caxias, RJ, Brasil.

${ }^{6}$ Instituto Nacional de Cardiologia Laranjeiras - Rio de Janeiro, RJ, Brasil.

Correspondência: Esmeralci Ferreira. Av. Alda Garrido, 84/cobertura - Barra da Tijuca - Rio de Janeiro, RJ, Brasil - CEP 22621-000

E-mail: ef.cardiol@terra.com.br

Recebido em: 9/1/2009 • Aceito em: 7/8/2009
}

\begin{abstract}
Acute Aortic and Left Main Coronary Dissection During Elective Coronary Stenting

Patient with post-infarction angina associated to severe lesions in the left circumflex artery and obtuse marginal branch was submitted to angioplasty with stenting, but developed a left main coronary artery (LMCA) dissection expanding to the ascending aorta, complicated with hypotension and cardiorespiratory arrest (CRA). She was successfully resuscitated but further hemodynamic instability developed and was resolved with LMCA stenting.
\end{abstract}

DESCRIPTORS: Aneurysm, dissecting. Angioplasty/adverse effects. Stents/adverse effects.

agudas, com isquemia, arritmias, choque e parada cardiorrespiratória.

\section{RELATO DO CASO}

Mulher de 70 anos, hipertensa, tabagista, dislipidêmica, com dor torácica de início súbito e evolução persistente. Foi internada com diagnóstico de infarto agudo do miocárdio inferior, com evolução que ultrapassava 12 horas. Realizou coronariografia nas primeiras 24 horas de internação, que demonstrou calcificações difusas e lesões críticas, tanto em artéria circunflexa, com $80 \%$ de obstrução proximal, como em artéria marginal esquerda, com $98 \%$ de obstrução ostial, onde se visualizava uma linha de dissecção com diminuição de fluxo (Figura 1), sendo esta última considerada a lesão culpada pelo evento agudo.

A estratégia de tratamento inicial foi a realização de angioplastia com stent Taxus ${ }^{\mathrm{TM}} 2,25 \times 20 \mathrm{~mm}$ no óstio da artéria marginal esquerda e stent Libertè ${ }^{\mathrm{TM}}$ $3,5 \times 16 \mathrm{~mm}$ na artéria circunflexa. O cateter-guia selecionado foi o $\mathrm{XB} 4^{\mathrm{TM}} 6 \mathrm{~F}$. A presença de grande tortuosidade no ramo da artéria marginal esquerda dificultou a passagem da lesão com a corda-guia 0,14" extrassuporte, e impediu a pré-dilatação com cateter- 
Ferreira E, et al. Dissecção Aguda de Aorta Ascendente e de Tronco de Coronária Esquerda Durante Implante Eletivo de Stents Coronários. Rev Bras Cardiol Invas. 2009;17(3):418-22.

balão Maverick ${ }^{\mathrm{TM}} 2 \times 15 \mathrm{~mm}$, mas foram realizadas pré-dilatações com o cateter-balão Maverick ${ }^{\mathrm{TM}} 2 \times 9 \mathrm{~mm}$ (Figura 2). O stent Taxus ${ }^{\mathrm{TM}} 2,25 \times 20$ mm não atravessou a lesão, nem mesmo com o auxílio de uma segunda corda-guia $0,14 "$, e esse segmento, tratado apenas com cateter-balão, permaneceu com lesão residual de $20 \%$, mas houve melhora do padrão de fluxo coronário.

Foi realizado, então, implante direto do stent Libertè $^{\text {TM }} 3,5 \times 16 \mathrm{~mm}$ na artéria circunflexa, com abertura da lesão, mas a injeção de controle após a retirada do cateter-balão revelou impregnação de contraste por dissecção da aorta ascendente (Figura 3). Na mesma injeção observamos diminuição do fluxo coronário para as artérias descendente anterior e circunflexa (fluxo TIMI II). A imagem de fluoroscopia mostrava dissecção de tronco de coronária esquerda. A pacien-

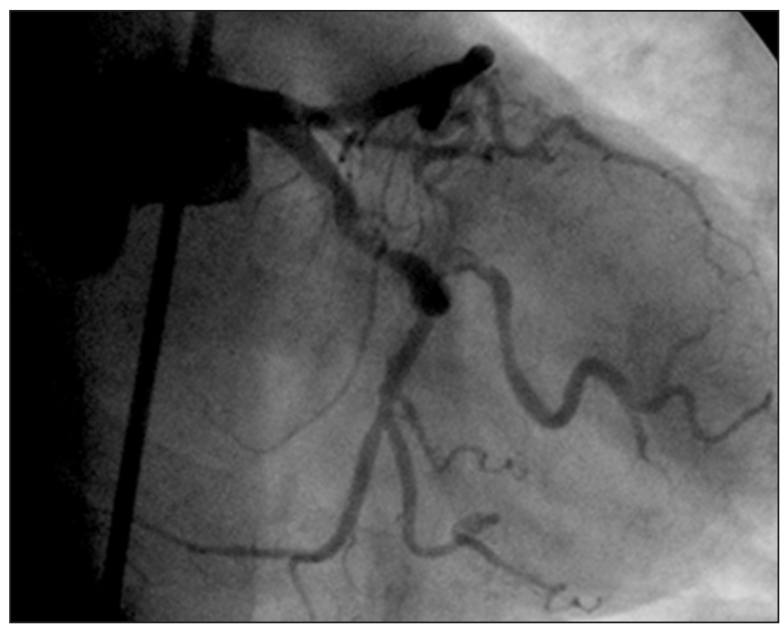

Figura 1 - Lesões obstrutivas na porção proximal da artéria circunflexa e óstio da artéria marginal esquerda. te apresentava dor constritiva intensa e supradesnível do segmento ST ao monitor, evoluindo com hipotensão grave e subsequente parada cardiorrespiratória por fibrilação ventricular. Foram iniciadas as manobras habituais de desfibrilação com sucesso, mas os níveis tensionais não melhoraram e a monitorização eletrocardiográfica evidenciava o mesmo padrão de supradesnível do segmento ST.

A cirurgia de emergência foi considerada como possível forma de resolução do quadro; entretanto, a rápida deterioração da condição hemodinâmica motivou a opção pelo implante imediato do stent Libertè ${ }^{T M}$ $4 \times 20 \mathrm{~mm}$, do segmento médio do tronco de coronária esquerda até a porção proximal da artéria circunflexa (Figura 4). Essa intervenção restabeleceu prontamente o fluxo coronário (inclusive na artéria descendente anterior) e selou a dissecção aórtica (Figuras 4 e 5), com melhora dos níveis tensionais, resolução da dor torácica e normalização do segmento ST ao monitor.

A paciente evoluiu assintomática nos cinco dias subsequentes, tendo declinado da realização de ultrassom intracoronário ou ecocardiograma transesofágico. O ecocardiograma transtorácico e o eletrocardiograma não demonstravam alterações evolutivas. A paciente persistia assintomática e mantinha o hábito de fumar no seguimento tardio após oito meses.

\section{DISCUSSÃO}

Historicamente, a incidência de dissecção iatrogênica da aorta ascendente varia de $0,02 \%$ a $0,08 \%$ em procedimentos diagnósticos, e entre $0,07 \%$ e $0,6 \%$ nas intervenções coronáriass ${ }^{1,2}$. Numa série recente de Gómez-Moreno et al. ${ }^{1}$, com 28.869 procedimentos diagnósticos e 12.031 intervenções coronárias, a dissecção iatrogênica da aorta ascendente se mostrou uma complicação rara, com incidência de 0,04\% com
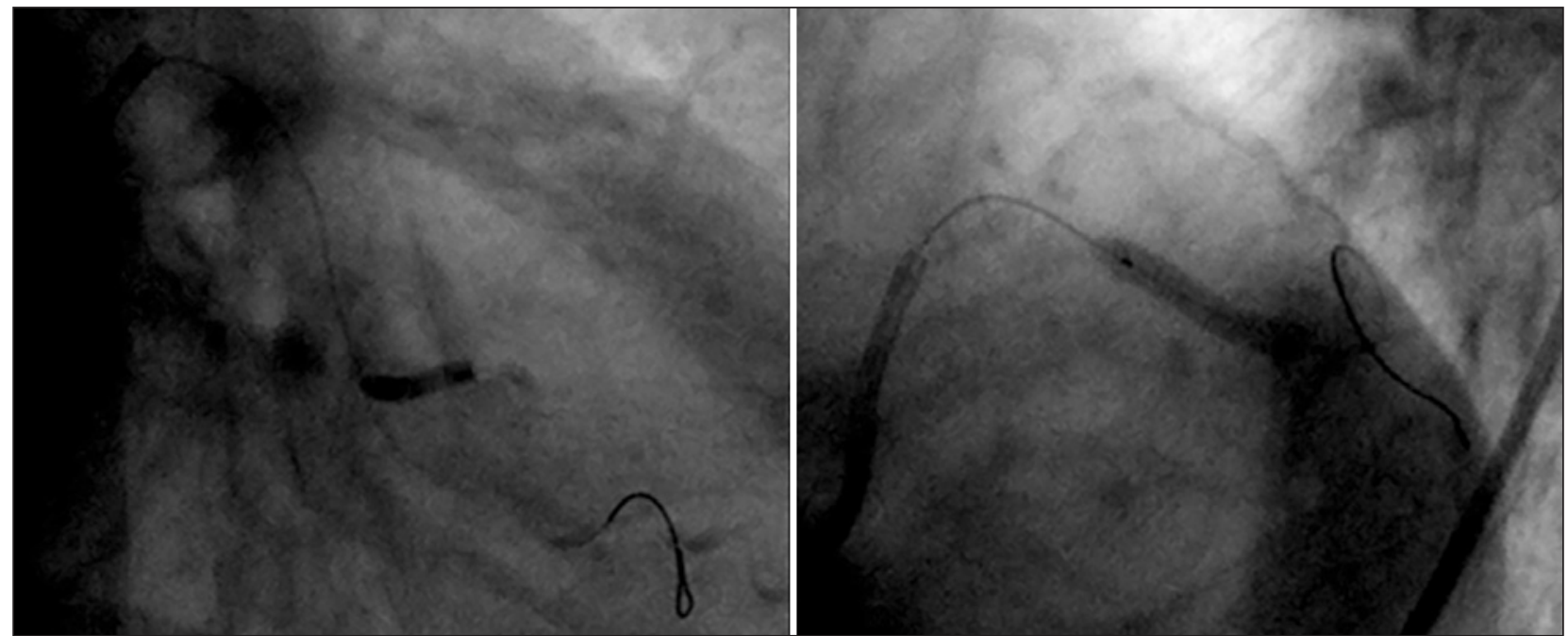

Figura 2 - À esquerda, pré-dilatação da lesão no ramo marginal; à direita, implante direto de stent na artéria circunflexa. 
Ferreira E, et al. Dissecção Aguda de Aorta Ascendente e de Tronco de Coronária Esquerda Durante Implante Eletivo de Stents Coronários. Rev Bras Cardiol Invas. 2009;17(3):418-22.

relação ao total de procedimentos, ainda que fosse significativamente maior nas intervenções terapêuticas $(0,01 \%$ vs. $0,12 \% ; P=0,0001)$.

A manobra mais frequentemente relacionada a essa complicação costuma ser a cateterização profunda da coronária (deep intubation), e nessa situação, especialmente quando se observa amortecimento da curva de pressão, a injeção de grandes quantidades de contraste deve ser evitada e o cateter, retirado ${ }^{3}$. Outras manobras comumente associadas são dilatação com balão, implante de stent e manipulação de cordas-guia ${ }^{1}$. Eventualmente a dissecção poderia ser precipitada por inapropriada inspiração profunda do paciente $^{4}$. A dissecção iatrogênica da aorta ascendente é mais frequente no contexto do infarto agudo do miocárdio ${ }^{2}$ e com o uso de cateteres não-convencio-

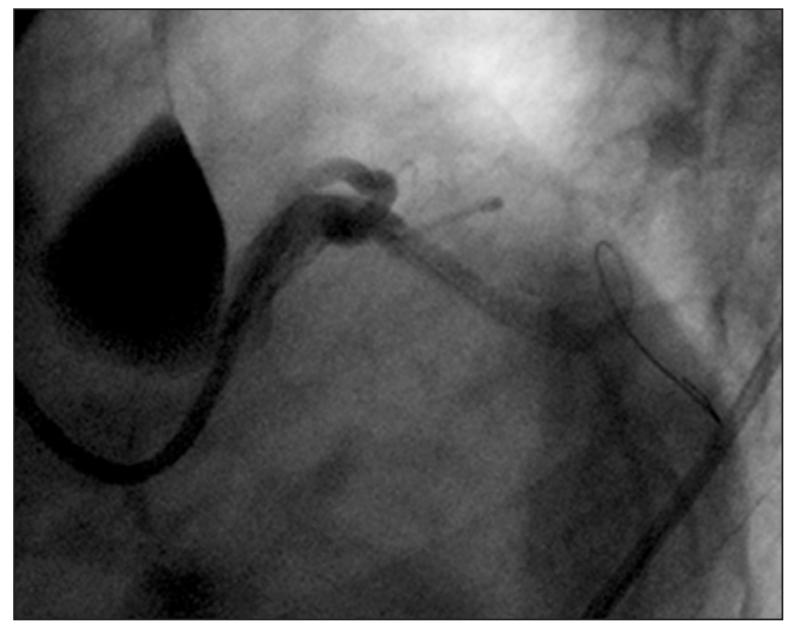

Figura 3 - Dissecção da aorta ascendente após implante de stent na artéria circunflexa.

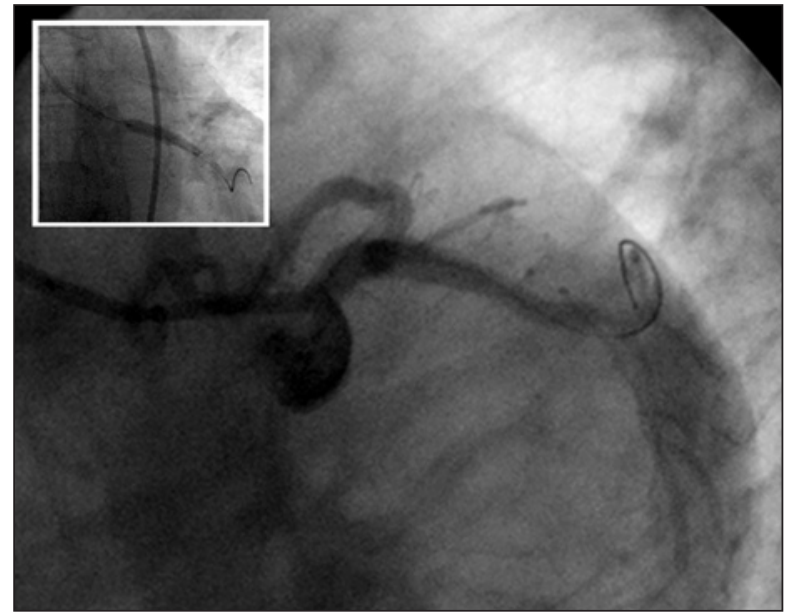

Figura 4 - Implante de stent no tronco da coronária esquerda com resolução da dissecção aórtica. Observa-se que o stent é dirigido para a artéria circunflexa. No destaque, detalhe do implante. nais (Amplatz ${ }^{T M}, X^{T M}, M^{T M}$ ), particularmente quando da manipulação da artéria coronária direita ou na recanalização de oclusões crônicas ${ }^{1,2,5,6}$.

Existem poucas informações a respeito da dissecção iatrogênica da aorta ascendente, mas sabe-se que sua apresentação e evolução clínica diferem da dissecção aórtica espontânea ${ }^{2}$. Ainda há controvérsias a respeito do manejo ideal, mas ele pode envolver um tratamento expectante, a intervenção percutânea isolada, o reparo cirúrgico ou uma combinação dessas estratégias $^{1,2,5}$.

$\mathrm{Na}$ descrição original de Alfonso et al. ${ }^{6}$, a conduta expectante se referia à não-realização de reparo aórtico, com relato de dois casos em que houve boa evolução. Em um deles houve implante de stent coronário e no outro, oclusão total da artéria coronária direita. São exemplos de que a dissecção coronária foi selada, impedindo a progressão da dissecção aórtica, por dois diferentes mecanismos ${ }^{6}$.

A classificação proposta por Dunning et al. ${ }^{2}$ (Tabela 1) leva em conta a extensão da lesão aórtica e se tornou consagrada ao mostrar que pacientes com menor envolvimento aórtico (classes I e II) tinham evolução bem favorável quando submetidos a implante de stent. Outros fatores, ainda mais importantes, são a existência ou não de dissecções anterógradas e/ou oclusões

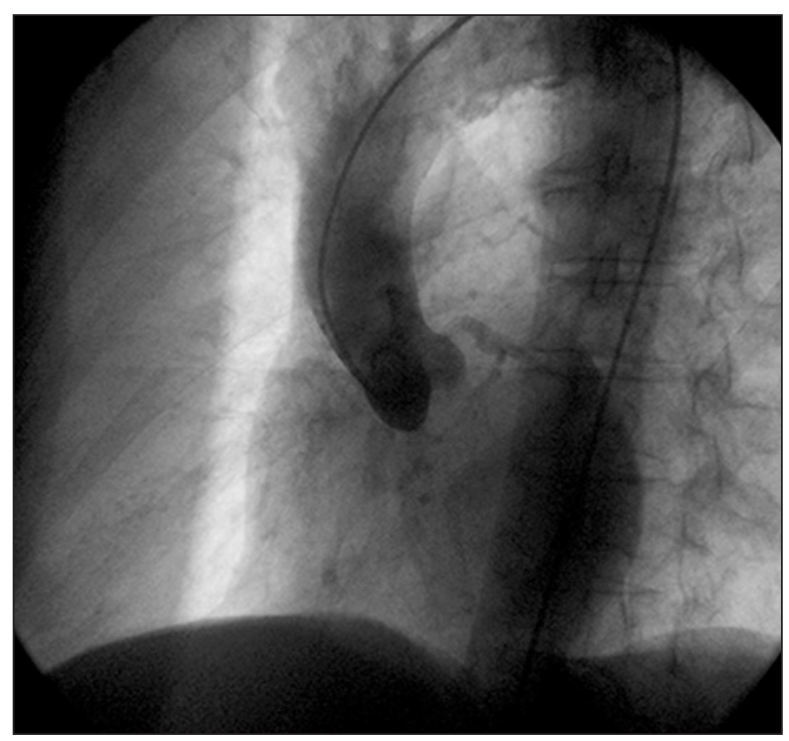

Figura 5 - Aortografia de controle sem sinais de dissecção.

TABELA 1 Classificação de Dunning ${ }^{2}$

Classe I Limitada ao seio de Valsalva correspondente

Classe II Estende-se a menos de 40 mm do arco aórtico

Classe III Estende-se a 40 mm ou mais do arco aórtico 
Ferreira E, et al. Dissecção Aguda de Aorta Ascendente e de Tronco de Coronária Esquerda Durante Implante Eletivo de Stents Coronários. Rev Bras Cardiol Invas. 2009;17(3):418-22.

coronárias associadas, bem como a presença de instabilidade hemodinâmica, que é uma clara indicação para intervenção ${ }^{5}$.

$\mathrm{Na}$ abordagem intervencionista, o fechamento do ponto de dissecção com o implante de stent pode ser realizado prontamente, e em cenários muitas vezes alarmantes. O implante imediato de stent, quando bemsucedido, converteria uma dissecção que evolui em hematoma intramural a ser reabsorvido ${ }^{1,2}$, evitando a realização de cirurgias de alto risco para reparo aórtico $^{1,2,6,7}$. Por esse motivo, essa forma de tratamento tem sido preconizada em todos os casos de intervenção, desde que o ponto de dissecção possa ser adequadamente identificado ${ }^{1,2,5-9}$.

Na série de Gómez-Moreno et al. ${ }^{1}$, foram identificados 18 pacientes com dissecção iatrogênica da aorta ascendente, dos quais 7 foram inicialmente submetidos a conduta expectante, não sendo realizada nenhuma intervenção. Todos tiveram evolução livre de eventos, pois eram dissecções limitadas ao seio de Valsalva (Dunning classe I), e 2 desses pacientes foram posteriormente submetidos a cirurgias eletivas para tratamento de doença coronária, sem necessidade de reparo aórtico. Nessa série, o ponto de entrada da dissecção só pôde ser visualizado angiograficamente em 10 pacientes (56\% dos casos), dado que mostra a importância da valorização das alterações de fluxo coronário e do entendimento dos mecanismos de dissecção associados ao instrumental na determinação do provável ponto de dissecção. Nesses 10 casos, o implante de stent levou a completa resolução, com reabsorção do hematoma intramural em 9 pacientes. A trombose de stent, com rápida deterioração hemodinâmica, levou à revascularização miocárdica de emergência em um paciente, sem reparo aórtico. Houve ainda um paciente com choque cardiogênico decorrente da dissecção de tronco de coronária esquerda com dissecção iatrogênica da aorta ascendente, que foi submetido a cirurgia de emergência como estratégia inicial e teve boa evolução. Esse paciente também não foi submetido a reparo aórtico ${ }^{1}$. Esses dados são consistentes com os de séries anteriores, em que a necessidade de cirurgias de urgência variava entre $22 \%$ e $25 \%{ }^{2,10}$. Em casos esporádicos, pensou-se em estabilizar a dissecção com implante de stents, ideia já utilizada em dissecções que envolvem a aorta torácica, abdominal ${ }^{11}$ ou mesmo seus ramos ${ }^{1}$, e nesses relatos não houve necessidade de cirurgia.

Nos casos em que se desenvolvem hematomas intramurais após o implante de stent, não está definida uma estratégia terapêutica, mas parece que aproximadamente $60 \%$ dos casos evoluem para reabsorção completa ${ }^{1}$. Há relatos de uso bem-sucedido de dupla terapia antiplaquetária e anticoagulação convencional nos casos em que houve implante de stent coronário e a dissecção foi selada ${ }^{12}$.
Após a dissecção coronária ter sido estabilizada, toda a atenção se volta para a extensão da dissecção aórtica. No laboratório de hemodinâmica, além da angiografia podem ser considerados o ultrassom intracoronário e o ecocardiograma transesofágico. Se a dissecção se mostra localizada e sem fluxo no falso lúmen, uma conduta expectante, com avaliação seriada por ecocardiograma transtorácico, ecocardiograma transesofágico, angiotomografia ou angiorressonância, pode ser mais apropriada ${ }^{1,12}$. Há relatos de dissecções limitadas ao seio de Valsalva (Dunning classe I) documentadas por ultrassom intracoronário e que não foram visualizadas ao ecocardiograma transtorácico nem à angiorressonância. O ultrassom intracoronário também se mostrou capaz de registrar o fechamento do ponto de entrada da dissecção ${ }^{1}$.

Se, por outro lado, há extensa dissecção aórtica, com propagação para os óstios coronários ou grandes vasos ou em associação com insuficiência aórtica, então a cirurgia deve ser considerada ${ }^{12,13}$. Outros fatores eventualmente associados ao prognóstico e à indicação cirúrgica, ainda que mais raros, são o grande diâmetro aórtico, a larga espessura do hematoma, e a presença de extravasamento de sangue no pericárdio ou na região periaórtica'.

A abordagem cirúrgica inicial tem ficado reservada para os casos em que o implante de stent não foi possível ou bem-sucedido; nesses casos, a cirurgia, quando realizada, apresenta resultados satisfatórios ${ }^{1,2,13}$. A revascularização miocárdica de emergência está indicada na presença de má perfusão coronária ou estenose residual grave. Outra indicação da cirurgia é a progressão da dissecção aórtica. Para alguns, a excisão parcial do seio coronário afetado com revascularização coronária seria preferível à prótese total aórtica. Outro ponto de interesse é a proteção miocárdica, já que a distribuição homogênea da solução de cardioplegia pode não ser possível pela infusão anterógrada na presença de dissecção coronária. Por isso, as infusões anterógrada e retrógrada combinadas podem ser preferíveis nesse contexto ${ }^{13}$. Em situações de urgência, a revascularização miocárdica pode ser realizada sem circulação extracorpórea, desde que a dissecção iatrogênica da aorta ascendente seja limitada e que a aorta não venha a ser abordada na cirurgia ${ }^{14}$. A estabilização do quadro clínico com balão intra-aórtico no préoperatório é considerada contraindicada na presença de dissecção aórtica ${ }^{5}$.

A melhor abordagem dessa grave complicação é seu conhecimento e prevenção, com escolha e manipulação adequadas do instrumental, aliados ao rápido reconhecimento da complicação por parte do operador $^{1,2,5}$. No caso descrito, a manipulação de diversos cateteres-balão e stent, em artérias calcificadas, provavelmente ocasionou a movimentação do cateter-guia e a consequente dissecção. Talvez a simples retirada de um cateter-balão, mesmo sem manobras intempes- 
Ferreira E, et al. Dissecção Aguda de Aorta Ascendente e de Tronco de Coronária Esquerda Durante Implante Eletivo de Stents Coronários. Rev Bras Cardiol Invas. 2009;17(3):418-22.

tivas, tenha ocasionado as dissecções retrógrada e anterógrada no tronco, envolvendo a aorta e a artéria circunflexa, respectivamente. A maioria dos intervencionistas experientes está apta a realizar rapidamente o implante de um stent no tronco da coronária esquerda. Entretanto, a completa restauração de lesão grave envolvendo a aorta é um fato que, além de técnica ou experiência, contou com a eventualidade do implante no ponto correto da dissecção, ainda que sua exata localização não fosse muito evidente.

\section{CONFLITO DE INTERESSES}

Os autores declararam inexistência de conflito de interesses.

\section{REFERÊNCIAS BIBLIOGRÁFICAS}

1. Gómez-Moreno $S$, Sabaté $M$, Jiménez-Quevedo $P$, Vázquez $P$, Alfonso F, Angiolillo DJ, et al. latrogenic dissection of the ascending aorta following heart catheterization: incidence, management and outcome. Eurolntervention. 2006;2:197-202.

2. Dunning DW, Kahn JK, Hawkins ET, O'Neill WW. Iatrogenic coronary artery dissections extending into and involving the aortic root. Catheter Cardiovasc Interv. 2000;51(4):387-93.

3. Meller J, Friedman S, Dack S, Herman MV. Coronary artery dissection - a complication of cardiac catheterization without sequelae: case report and review of the literature. Cathet Cardiovasc Diagn. 1976;2(3):301-8.

4. Philippe F, Bouabdallah K, Dibie A, Laborde F. Left main artery dissection during 4 French coronary angiography in elderly patient with severe aortic stenosis: "speedy recovery" using emergency primary stenting. J Interv Cardiol. 2002;15(3): 219-21.
5. Awadalla H, Salloum JG, Smalling RW, Sdringola S. Catheterinduced dissection of the left main coronary artery with and without extension to the aortic root: a report of two cases and a review of the literature. J Interv Cardiol. 2004;17(4):253-7.

6. Alfonso F, Almería C, Fernández-Ortíz A, Segovia J, Ferreirós J, Goicolea J, et al. Aortic dissection occurring during coronary angioplasty: angiographic and transesophageal echocardiographic findings. Cathet Cardiovasc Diagn. 1997;42(4):412-5.

7. Carstensen S, Ward MR. latrogenic aortocoronary dissection: the case for immediate aortoostial stenting. Heart Lung Circ. 2008;17(4):325-9.

8. Al-Saif SM, Liu MW, Al-Mubarak N, Agrawal S, Dean LS. Percutaneous treatment of catheter-induced dissection of the left main coronary artery and adjacent aortic wall: a case report. Catheter Cardiovasc Interv. 2000;49(1):86-90.

9. Mulvihill NT, Boccalatte M, Fajadet J, Marco J. Catheterinduced left main dissection: a treatment dilemma. Catheter Cardiovasc Interv. 2003;59(2):214-6.

10. Januzzi JL, Sabatine MS, Eagle KA, Evangelista A, Bruckman $\mathrm{D}$, Fattori $\mathrm{R}$, et al. latrogenic aortic dissection. Am J Cardiol. 2002;89(5):623-6.

11. Gorog DA, Watkinson A, Lipkin DP. Treatment of iatrogenic aortic dissection by percutaneous stent placement. J Invasive Cardiol. 2003;15(2):84-5.

12. Goldstein JA, Casserly IP, Katsiyiannis WT, Lasala JM, Taniuchi M. Aortocoronary dissection complicating a percutaneous coronary intervention. J Invasive Cardiol. 2003;15(2):89-92.

13. Yilik L, Lafci B, Özsöyler I, Emrecan B, Gürbüz A. Surgical treatment of aortic dissection occurring during coronary angioplasty: a report of two cases. Temmuz. 2007;15(3): 238-41.

14. Bapat VN, Venn GE. A rare case of aortocoronary dissection following percutaneous transluminal coronary angioplasty: successful treatment using off-pump coronary artery bypass grafting. Eur J Cardiothorac Surg. 2003;24(2):312-4. 